Check for updates

Cite this: RSC Adv., 2019, 9, 23535

\title{
Water vapor permeable polyurethane films based on the hyperbranched aminoethers of boric acid
}

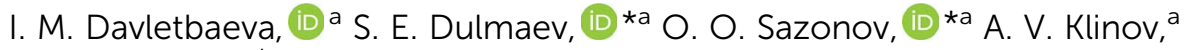 \\ R. S. Davletbaev ${ }^{\text {and A. M. Gumerov }}{ }^{\mathrm{a}}$
}

The hyperbranched polymers have drawn intensive attention in the design of macromolecules and functional materials because of their unique physical and chemical properties resulting from the branched architecture and the high number of functional groups. In the present study, by means of light scattering, viscosimetry, thermomechanical analysis, tensile stress-strain, mechanical loss tangent and water vapor permeability measurements, we demonstrate the hierarchical macromolecular organization of organoboron polyurethanes synthesized using sterically hindered amino ethers (AEBA) of boric acid. It is shown that the water vapor permeability of polyurethanes obtained on the basis of sterically hindered aminoethers of boric acid is due to the peculiarities of the chemical structure of AEBA, which can exhibit an ionomeric nature and the presence of steric hindrances created in the hyperbranched structure of AEBA, which can lead to an increase in free volume in such polyurethanes.

Received 11th July 2019

Accepted 25th July 2019

DOI: 10.1039/c9ra05314j

rsc.li/rsc-advances increase. Hayashi et al. ${ }^{4}$ prepared PUs with different molecular weight polyols and showed that the water vapor permeability of the PU membranes depends upon the ethylene oxide concentration. The effects of the block length of PEG on membrane mass transfer through PU materials were investigated by $\mathrm{Hu}$ and Mondal. ${ }^{5}$ They prepared hydrophilic PUs and their results showed that sorption and water vapor permeability of the materials increased linearly with increasing PEG block length and temperature.

A series of thermosensitive PU films for high temperature applications were synthesized by Morel et al. ${ }^{6}$ and it was found that the water vapor permeability is related to the crystallization of the soft segments. Hsieh et al. ${ }^{7}$ noticed that the permeability of a PU membrane decreases with the crystallinity of soft segments, as vapor is transferred only through amorphous regions. Since the properties of segmented PUs are defined by the presence of their amorphous or crystalline structure, the mobility of the soft segments increases and promotes the moisture diffusion. ${ }^{8-14}$

Summarizing the results of the researchers described above, a common feature that determines the efficiency of vapor permeability of segmented PUs is the existence of free volume. The increase in free volume leads to an increase in the vapor permeability of PU membranes. At the same time, the possibilities for increasing the free volume of polymeric materials are associated with polymers obtained on the basis of hyperbranched structures.

Since Flory's theoretical report, ${ }^{15}$ hyperbranched polymers (HBPs) have been emerging at the cutting edge of polymer chemistry and material science as catalysts, ${ }^{\mathbf{1 6 - 2 3}}$ advanced
${ }^{a}$ Kazan National Research Technological University, 68 Karl Marx Str., Kazan, Republic of Tatarstan 420015, Russian Federation. E-mail: davletbaeva09@mail.ru; impsble@gmail.com; sazonov.oleg2010@gmail.com

${ }^{b}$ Kazan National Research Technical University named after A.N.Tupolev-KAI, 10 Karl Marx Str., Kazan, Republic of Tatarstan 420111, Russian Federation 
materials, ${ }^{24-33}$ as well as for biomedical purposes. ${ }^{34-39}$ Being the class of polymers with irregular topological structure, HBPs consist of randomly distributed dendritic, linear and terminal units. Branching points together with numerous terminal groups give to such polymers the unique physical and chemical properties such as low melt viscosity, high solubility, tunable crosslinking degree, polarity, crystallinity, ${ }^{40}$ etc. Currently, the class of HBPs has received increasing interest in many fields including gas separation ${ }^{41-47}$ and barrier coatings. ${ }^{48}$ In fact, transport of gases through polymeric membranes may be explained by the "free volume" model, according to which the diffusion rate of different gases depends on the presence of microcavities among polymer chains. ${ }^{49}$ In this sense, highly branched macromolecular architecture results are totally different from conventional linear polymers intermolecular packing that may enhance gas transport properties.

Recently, a permeability of various gases across hyperbranched polymeric membranes was investigated in a number of works explicating their gas separation behavior. So, Suzuki et $a l .^{50}$ synthesized hyperbranched polyimide membranes, which exhibited lower permeabilities compared with linear ones, but higher $\mathrm{O}_{2} / \mathrm{N}_{2}$ and $\mathrm{CO}_{2} / \mathrm{N}_{2}$ selectivities. In another work, the diaminobutane dendrimers were employed by Shao et $a l .{ }^{51}$ as cross-linking agents for the polyimide membranes, which exhibited superior gas separation performance compared to the traditional trade-off line. Kovvali and Sirkar introduced the polyamidoamine dendrimers into liquid membranes to facilitate $\mathrm{CO}_{2}$ separation. ${ }^{52}$ In the study by Yao, the flexible microporous hyperbranched polyimides were tested as carbon dioxide adsorbents, which demonstrated gas uptake and adsorption behavior comparable to that of some other microporous polyimides. ${ }^{47}$ In order to achieve synergetic enhancement of the permselectivity of linear polyimides, Suzuki et al. have modified a membrane composition introducing hyperbranched polyimides and silica. ${ }^{53}$ Hydroxy-functional hyperbranched aliphatic polyesters were evaluated for use as barrierimproving additives on flexible packaging materials against oxygen and water vapor. ${ }^{54}$ It was found that under humid conditions the highest barrier properties are observed for coatings containing a minimal content of hydroxyl groups. Finally, the effect of HBP on the shape memory of the thiol-ene polymers was studied by Jeong and Kim. ${ }^{55}$

In our previous works, ${ }^{56,57}$ the polyurethanes based on hyperbranched amino ethers of boric acid (AEBA) were synthesized in order to create the novel membrane materials with high values of gas permeability. Aimed to introduce hydrolytically stable B-O-C moieties into a polymeric framework, the approach of sterically hindered hyperbranched structures formation was proposed to hamper the interactions with water molecules. Indeed, the polymeric materials obtained in this manner were stable under humid conditions and exhibited the relatively high separation toward the polar gases.

In continuation of previous works, the present study intends to investigate the effect of steric hindrance substituents in the hyperbranched structure of the amino ethers of boric acid on the main causes leading to water vapor permeability of the resulting PU membranes.

\section{Experimental}

\subsection{Materials}

PEG was purchased from PJSC Nizhnekamskneftekhim (Nizhnekamsk, Russia). Triethanolamine (TEA), diethanolamine (DEA) and monoethanolamine (MEA) were obtained from OJSC Kazanorgsintez (Kazan, Russia). Boric acid (99.99\%) was purchased from Sigma-Aldrich. The epoxy resin - diglycidyl ether of 4,4'-dihydroxy-2,2-diphenylpropane (ED-20) was obtained from JSC Chimexltd (St. Petersburg, Russia). Polyisocyanate (PIC) "Wannate PM-200" was purchased from Kumho Mitsui Chemicals, Inc. (China). Octaglycidyl polyhedral oligomeric silsesquioxane (Gl-POSS) was purchased from Hybrid Plastics (Hattiesburg, USA). GL-POSS is unstable over time and, therefore, it was closed tightly and stored in a cool, dry place at $10^{\circ} \mathrm{C}$.

PEG was additionally dried at $1-3 \mathrm{~mm} \mathrm{Hg}$ and $90{ }^{\circ} \mathrm{C}$ down to $0.01 \%$ of moisture concentration.

\subsection{Synthetic procedures}

General procedure for the synthesis of amino ethers of boric acid. Amino ethers of boric acid were obtained in two stages. In the first stage (according to the molar ratio of [TEA]: $\left[\mathrm{H}_{3} \mathrm{BO}_{3}\right]$ $:[\mathrm{PEG}]=1: 6: 11)$, boric acid (6 mol-2.793 g), PEG (3 mol-9.036 $\mathrm{g}$ ) and calculated amount of adduct (wt\%) were added to $250 \mathrm{~mL}$ three-necked round-bottom flask. In the first stage, the mixture was heated to $363 \mathrm{~K}$ for 2 hours under residual pressure $(10 \mathrm{~mm}$ $\mathrm{Hg}$ ). In the second stage, the remaining PEG (8 mol-24.096 g) and TEA ( 1 mol-1.124 $\mathrm{g}$ ) were added to the first mixture, followed by heating under similar conditions. The synthesized liquid AEBAPEG was collected into the stoppered flask.

The reaction was quenched after reaching the desired amount of hydroxylation toward the target product. Reaction progress was monitored by titration to determine hydroxyl group concentration.

General procedure for the synthesis of the adducts. The adduct based on the one-step reaction of ED-20, MEA and DEA (EMD) was obtained in one stage.

To prepare the EMD adduct, the following components were used: ED-20, MEA and DEA at their molar ratio of $1: 1: 1$. Ethyl acetate was used as a solvent. Synthesis was carried out in one stage. The calculated amount of ED-20 (1 mol-5.616 g), DEA (1 mol-1.735 g), MEA (1 mol-1.012 g) and ethyl acetate was introduced into a two-necked flask, heated under reflux to $T=$ $78{ }^{\circ} \mathrm{C}$ and held for two hours. At the end of the synthesis, it was poured into a container and the solvent was removed.

The adduct based on the one-step reaction of Gl-POSS and DEA (DEA-Gl-POSS) was obtained in one stage.

To prepare the DEA-Gl-POSS adduct, the following components were used: Gl-POSS and DEA at their molar ratio of $1: 8$. Toluene was used as a solvent. Synthesis was carried out in one stage. The calculated amount of Gl-POSS (1 mol-5 g), DEA (8 mol-3.431 g) and toluene was introduced into a two-necked flask, heated under reflux to $T=78{ }^{\circ} \mathrm{C}$ and held for two hours. At the end of the synthesis, it was poured into a container and the solvent was removed. 
General procedure for the synthesis of PU films based on amino ethers of boric acid. Polymeric films were obtained by bulk polymerization. The synthesized AEBA was mixed with PIC in a ratio of $1: 1$ to obtain the PU films. The polymerization was carried out on a glass surface. The cast film was dried in the vacuum oven at $80{ }^{\circ} \mathrm{C}$ for two hours. The flat-sheet polymeric films with a diameter equal to $80 \mathrm{~cm}$ were obtained and used for further investigation.

\subsection{Dynamic light scattering (DLS)}

DLS experiments were carried out on the Zetasizer Nano ZS (Malvern, Great Britain). This instrument has a $4 \mathrm{mV} \mathrm{He}-\mathrm{Ne}$ laser, which works on the $632.8 \mathrm{~nm}$ wavelength. Measurements were carried out at the $173^{\circ}$ detection angle. The experiments were carried out at $25{ }^{\circ} \mathrm{C}$ in the disposable plastic cuvettes of $1 \mathrm{~cm}$ path length.

\subsection{Viscosity measurements}

The dynamic viscosity of the samples was determined at the temperature range from $20^{\circ} \mathrm{C}$ to $80^{\circ} \mathrm{C}$, at atmospheric pressure and using a SVM 3000 Stabinger Viscometer (Anton Paar, Austria) with an error of $0.1 \%$. At the same time, the density determination was carried out with an error of $0.0002 \mathrm{~g} \mathrm{~cm}^{-3}$.

\subsection{Water vapor permeability (WVP) measurements}

WVP was measured according to the ASTM method of E 96-80B. Round mouth cylindrical glass cups with diameter of $50 \mathrm{~mm}$ and a height of $70 \mathrm{~mm}$ were filled with deionized water. Membranes were placed over the top of the cups and secured the perfect sealing between the cup and the membranes. The gaps between the membrane and the water surface were about $15 \mathrm{~mm}$. The cups were placed in a constant temperature chamber at the temperature $22{ }^{\circ} \mathrm{C}$ and $40{ }^{\circ} \mathrm{C}$. The weight losses after $24 \mathrm{~h}$ were measured. The result of water vapor permeability was calculated by the following formula:

$$
\mathrm{WVP}=\frac{G}{t A}
$$

where $G$ is the weight change in grams; $t$ is the duration of the test in hours; $A$ is the test area in $\mathrm{m}^{2}$.

During all WVP measurements, air surrounding the membranes had a constant temperature and relative humidity of $70 \%$. Sample thicknesses for all measurements were in the range of approximately 120 microns. On an average, three different readings were used for each WVP measurement, which were expressed in units of $\mathrm{g} \mathrm{m}^{-2} 24 \mathrm{~h}$.

\subsection{Tensile stress-strain measurements}

Tensile stress-strain measurements were obtained from the film samples of size $40 \mathrm{~mm} \times 15 \mathrm{~mm}$ with Universal Testing Machine Inspekt mini (Hegewald \& PeschkeMeß- und Prüftechnik GmbH, Nossen, Germany) at $293 \pm 2 \mathrm{~K}, 1 \mathrm{kN}$. The crosshead speed was set at $50 \mathrm{~mm} \mathrm{~min}^{-1}$ and the test continued until sample failure. Minima of five tests were analyzed for each sample and the average values were reported.

\subsection{Thermomechanical analysis (TMA)}

The thermomechanical curves of polymer samples were obtained using the TMA $402 \mathrm{~F}$ thermomechanical analyzer (Netzsch, Selb, Germany) in the compression mode. The sample thickness was $2 \mathrm{~mm}$, and the rate of heating was $3{ }^{\circ} \mathrm{C} \mathrm{min}{ }^{-1}$ in the static mode. The load was $2 \mathrm{~N}$.

\subsection{Mechanical loss tangent measurements (MLT)}

The MLT curves of polymer samples were taken using the dynamic mechanical analyzer DMA 242 (Netzsch, Selb, Germany) in the mode of an oscillating load. Force and stress-strain correspondences were calibrated using a standard mass. The thickness of the sample was $2 \mathrm{~mm}$. Viscoelastic properties were measured under nitrogen. The samples were heated at the rate of $3{ }^{\circ} \mathrm{C} \min ^{-1}$ and frequency of $1 \mathrm{~Hz}$. MLT was defined as the ratio of the viscosity modulus $G^{\prime \prime}$ to the elasticity modulus $G^{\prime}$.

\section{Results and discussion}

\subsection{Synthesis and characterization of amino ethers of boric acid modified by adduct EMD}

To prepare hyperbranched AEBA, triethanolamine (TEA) was used as the center of branching, and boric acid as the main element of AEBA construction. An adduct (EMD) based on the one-stage interaction of diglycidyl ether of 4,4'-dihydroxy-2,2diphenylpropane with DEA and MEA (Scheme 1) was obtained to create steric hindrances in the AEBA-PEG composition. Then, on the basis of AEBA-PEG, the corresponding aminoethers of boric acid AEBA-EMD (Scheme 2) were obtained. This scheme shows only an estimated location of the interaction of the $\mathrm{B}-\mathrm{OH}$ bond with the EMD, since it is not possible to accurately determine a location of a particular attachment (Scheme 3).

In previous studies, ${ }^{56,57}$ aminoethers of boric acid synthesized with use of triethylene glycol (TEG) were investigated. By the interaction of 1 mole of TEA, 3 moles of $\mathrm{H}_{3} \mathrm{BO}_{3}$ and 6 moles of TEG, AEBA-3 was obtained, and on the basis of 1 mole of TEA, 6 moles of $\mathrm{H}_{3} \mathrm{BO}_{3}$ and 12 moles of TEG, AEBA- 6 was obtained. It was shown that in the case of AEBA-3 and AEBA-6, the formation of intermolecular complexes occurs. With an increase in the size of the glycol component, the probability of their formation decreases.

According to measurements of the size distribution of particles in terms of intensity (Fig. 1a), AEBA-3 and AEBA-6 form large cluster unions. The average cluster size for AEBA-3 is $600 \mathrm{~nm}$, and for AEBA-6 it grows to $1000 \mathrm{~nm}$.

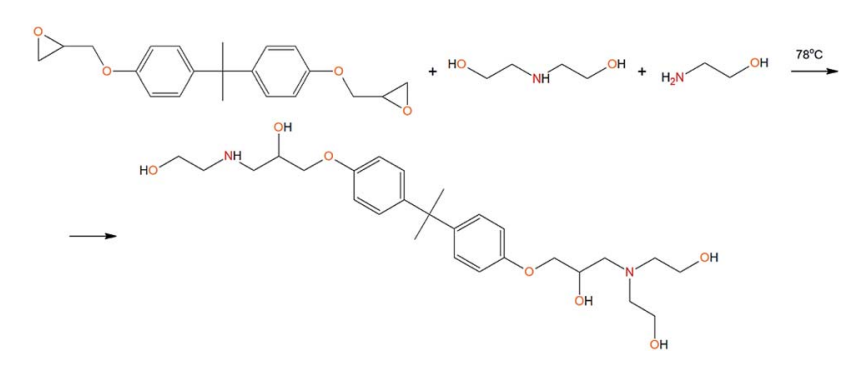

Scheme 1 Synthesis of EMD. 


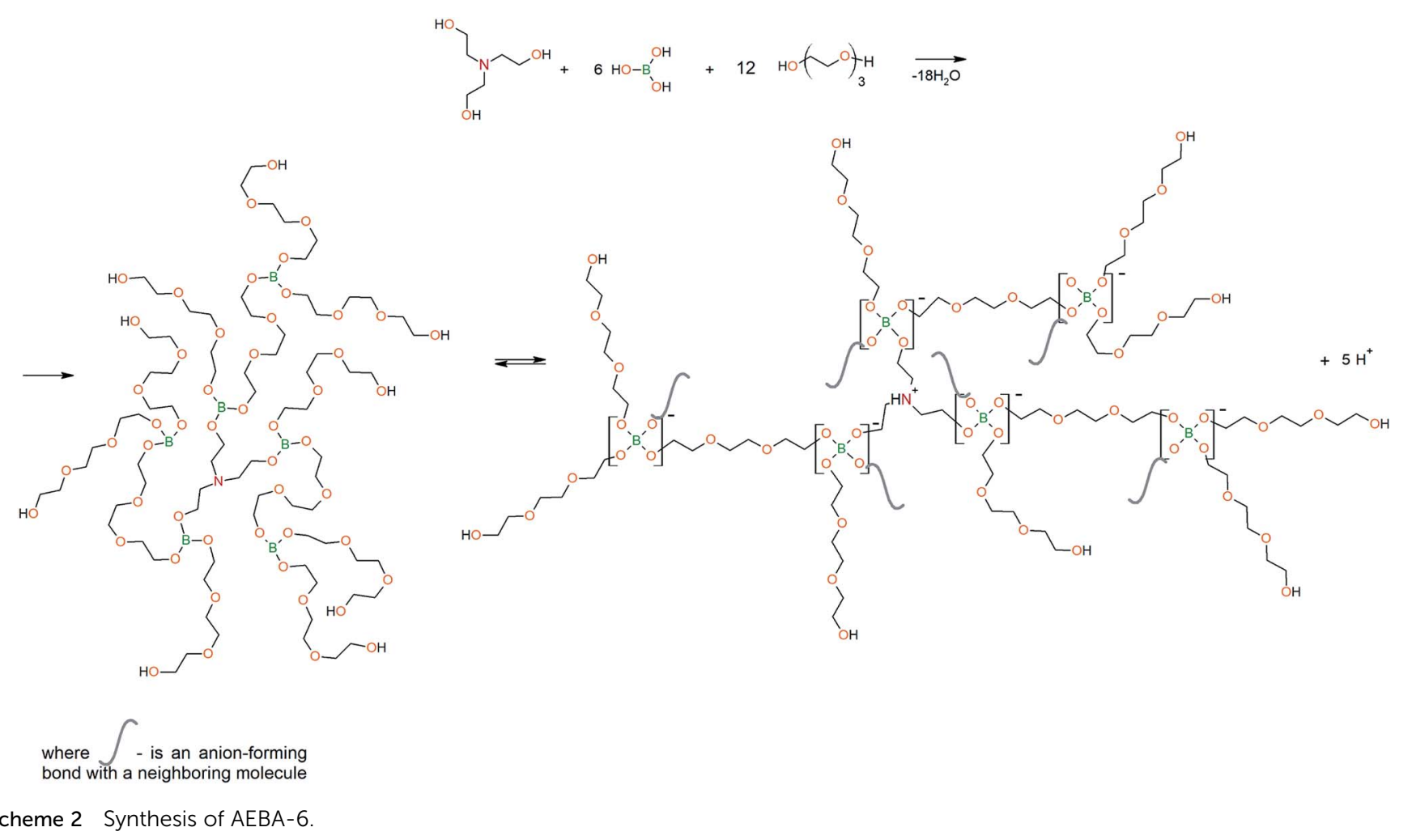

Due to the fact that PEG has a higher molecular weight in comparison with TEG, the probability of the participation of AEBA-PEG in the formation of intermolecular complexes noticeably decreased. As a result, AEBA-PEG sizes (250 nm) turned out to be almost three times smaller than AEBA- 6 sizes (Fig. 1b). The use of small amounts of EMD creates even greater obstacles to the formation of intermolecular complexes and leads to a decrease in the size of AEBA-EMD-1.0 to $150 \mathrm{~nm}$. In the case of AEBA-EMD-0.5 and AEBA-EMD-1.0 the particle size distribution turned out to be narrower (Fig. 1b).

However, despite the fact that the AEBA-EMD sizes turned out to be smaller compared to the AEBA- 3 and AEBA- 6 sizes, the particle size values of AEBA-EMD none the less remained large. In this regard, it can be concluded that AEBA-EMD, similarly to AEBA-3 and AEBA-6 (Scheme 4), participates in intermolecular interactions accompanied by the formation of borate anions and a tertiary ammonium cation.

An analysis of the density for AEBA-EMD (Table 1) allows us to conclude that the introduction of large hydroxyl-terminated molecules into the AEBA-PEG structure leads to changes in the spatial packaging of hyperbranched aminoethers of boric acid.

According to measurements of the dependence of the dynamic viscosity of AEBA-EMD on the EMD content (Fig. 2), the viscosity of AEBA-EMD almost does not change with increasing content of EMD in comparison with the viscosity of AEBA-PEG in the temperature range up to $30{ }^{\circ} \mathrm{C}$. As the temperature rises, the dynamic viscosity of AEBA-EMD becomes slightly lower compared to AEBA-PEG. The high dynamic viscosity values of AEBA-EMD compared to PEG confirm the

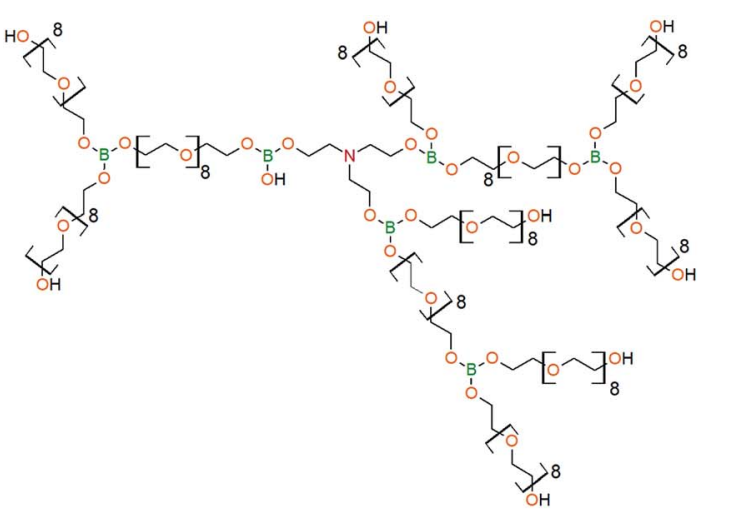

Scheme 3 Synthesis of AEBA-EMD.

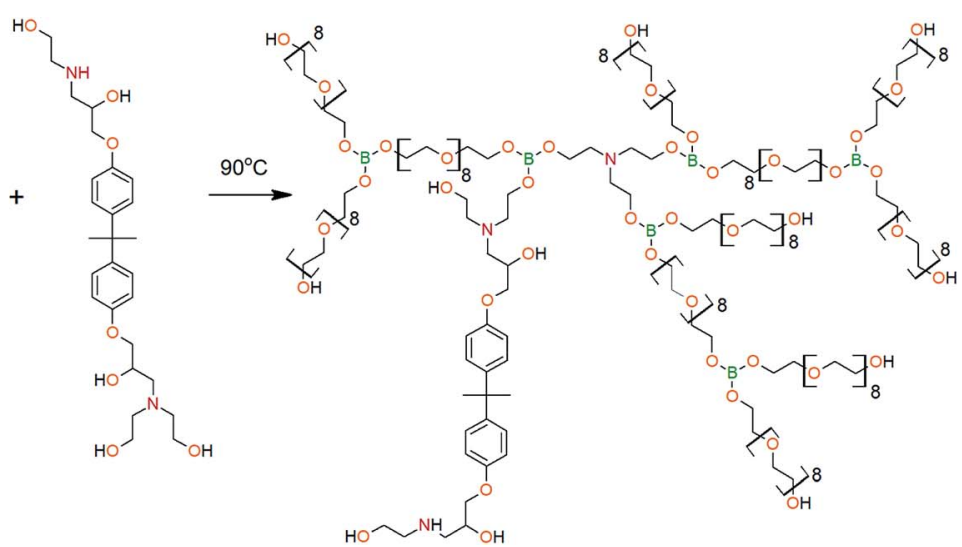



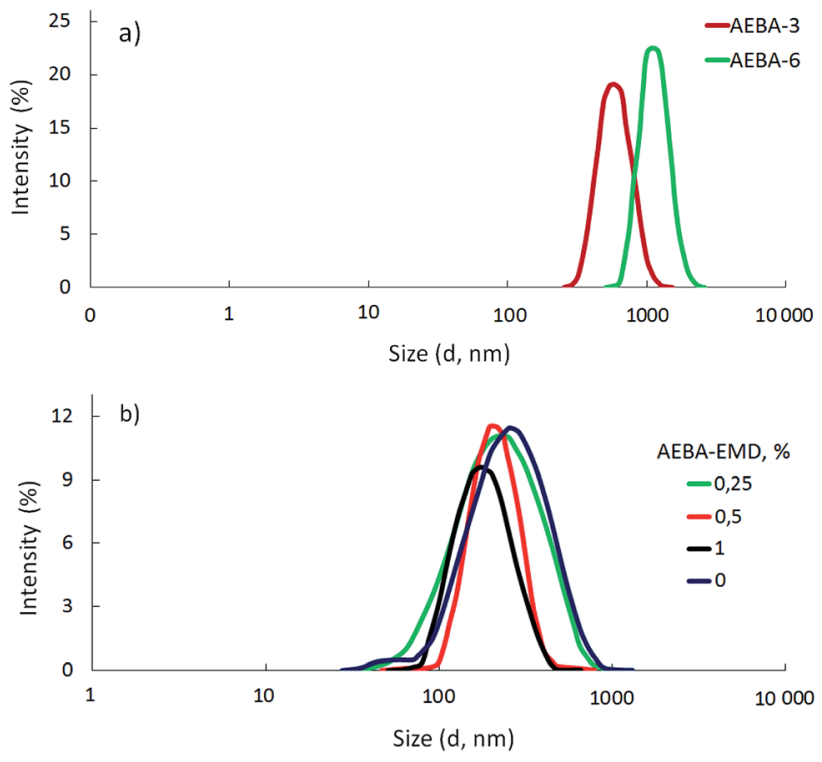

Fig. 1 Particle size distribution of (a) AEBA-3, AEBA-6 and (b) AEBAPEG-EMD, determined by DLS.

findings from DLS measurements. According to the fact that viscosity of hyperbranched polymers and oligomers are characterized by low viscosity compared with linear analogues, ${ }^{58,59}$ it can be concluded, that the reason for the high viscosity of AEBAEMD is due to the existence of network of intermolecular bonds.

\subsection{PU films based on hyperbranched amino ethers of boric acid modified by adduct EMD}

AEBA-EMD were used as a hydroxyl-terminated hyperbranched base for the synthesis of PU films.

For film samples of AEBA-EMD-PU (Fig. 3), the values of WVP at $22{ }^{\circ} \mathrm{C}$ depend little on the EMD content. With an increase in temperature to $40{ }^{\circ} \mathrm{C}$, a noticeable increase in the values of WVP is observed. In this case, the use of AEBA-EMD for the synthesis of the corresponding PU leads to a twofold increase in WVP in
Table 1 Density of AEBA-EMD

\begin{tabular}{|c|c|}
\hline EMD content, wt $\%$ & $\rho, \mathrm{g} \mathrm{cm}^{-3}$ \\
\hline 0 & 1.1434 \\
\hline 0.25 & 1.1515 \\
\hline 0.5 & 1.1483 \\
\hline 1 & 1.1513 \\
\hline 1.5 & 1.1503 \\
\hline 1.75 & 1.1482 \\
\hline 2 & 1.1484 \\
\hline 2.25 & 1.1487 \\
\hline
\end{tabular}

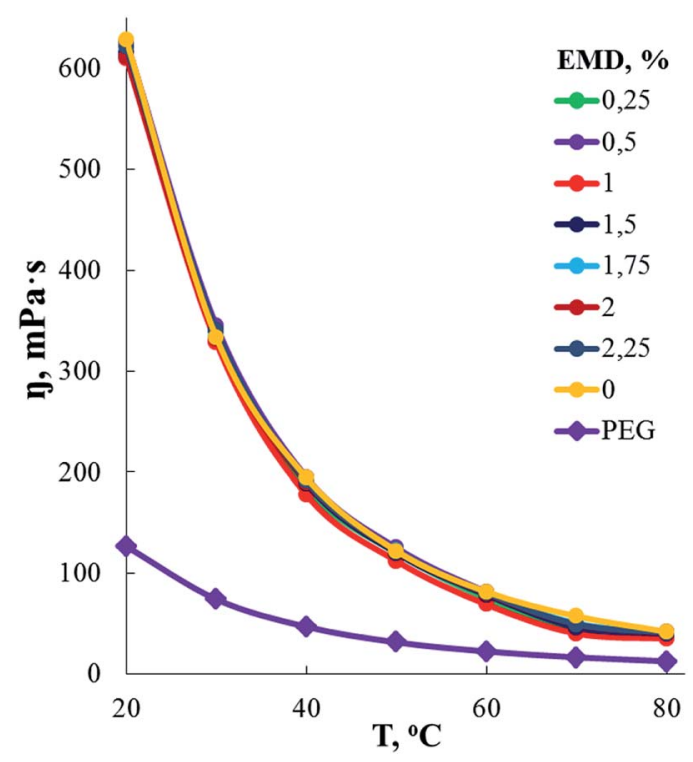

Fig. 2 Temperature dependencies of dynamic viscosity ( $\eta, \mathrm{mPa} s)$ of AEBA-EMD.

comparison with the unmodified AEBA-PEG-PU. With an increase in the EMD content of AEBA-EMD to $2.25 \mathrm{wt} \%$, WVP can reach values of $1820 \mathrm{~g} \mathrm{~m}^{-2}$ for 24 hours at $40{ }^{\circ} \mathrm{C}$. The ratio
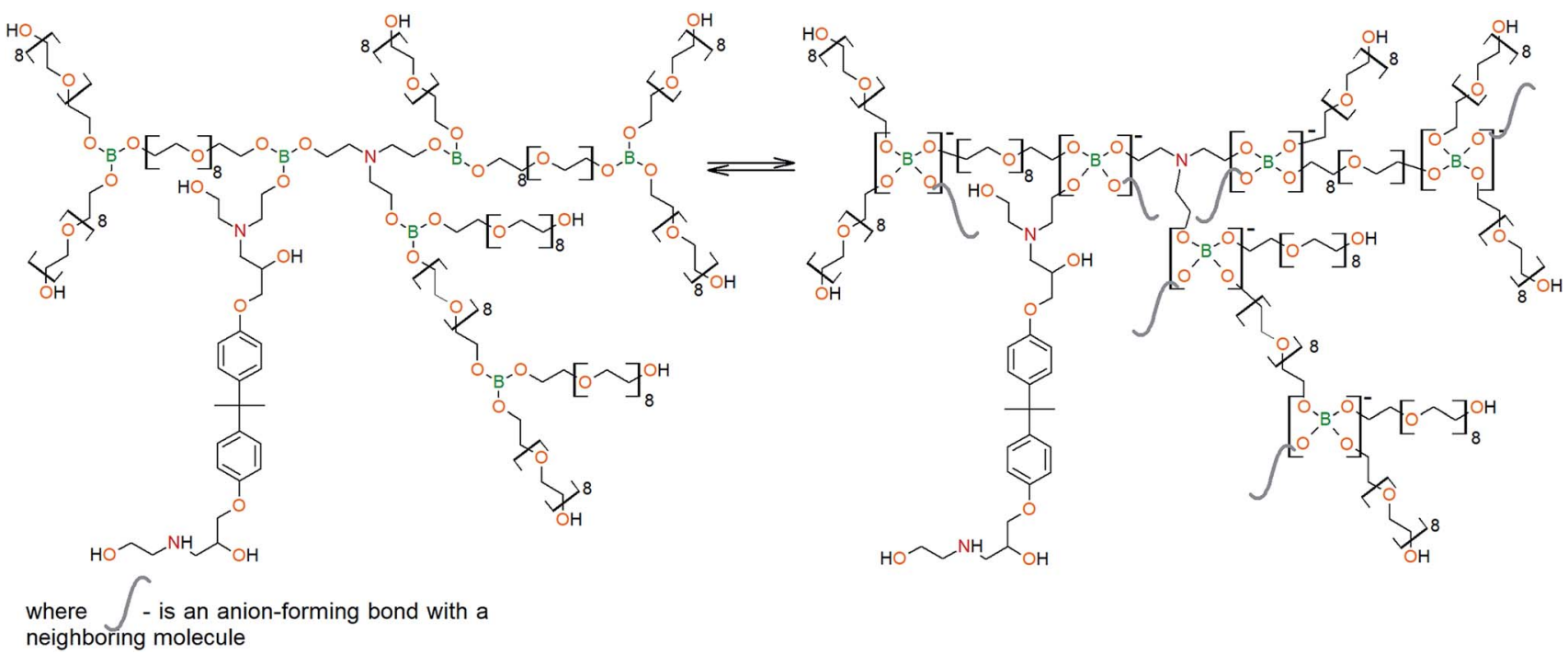

Scheme 4 Intermolecular interaction and complex formation in AEBA-EMD. 


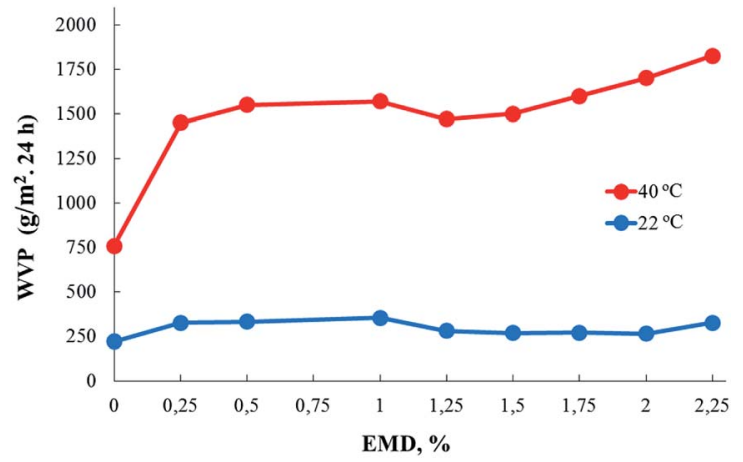

Fig. 3 Dependence of WVP for AEBA-EMD-PU on the content of EMD (C, \%).
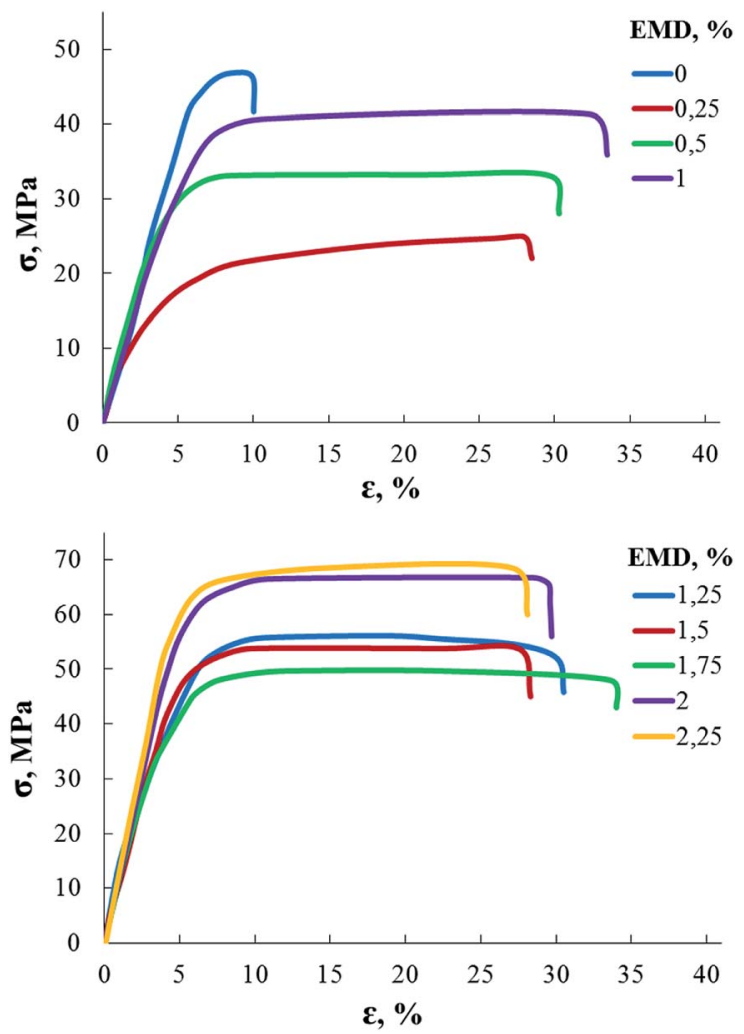

Fig. 4 Tensile tests of AEBA-EMD-PU.

Table 2 Young's modulus of AEBA-EMD-PU

EMD content, wt $\%$ Young's modulus, MPa

0

0.25

0.5

1

1.25

1.5

1.75

2

2.25
898

703

842

980

1233

1101

1170

1480

1560
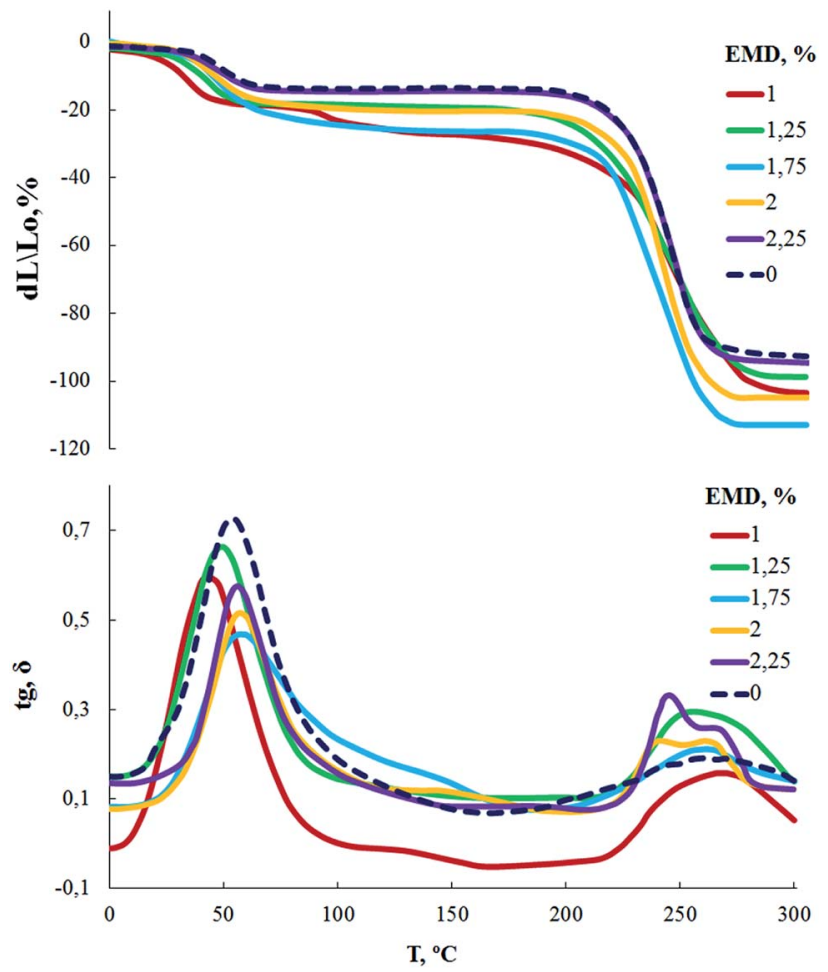

Fig. 5 TMA and MLT curves of AEBA-EMD-PU.

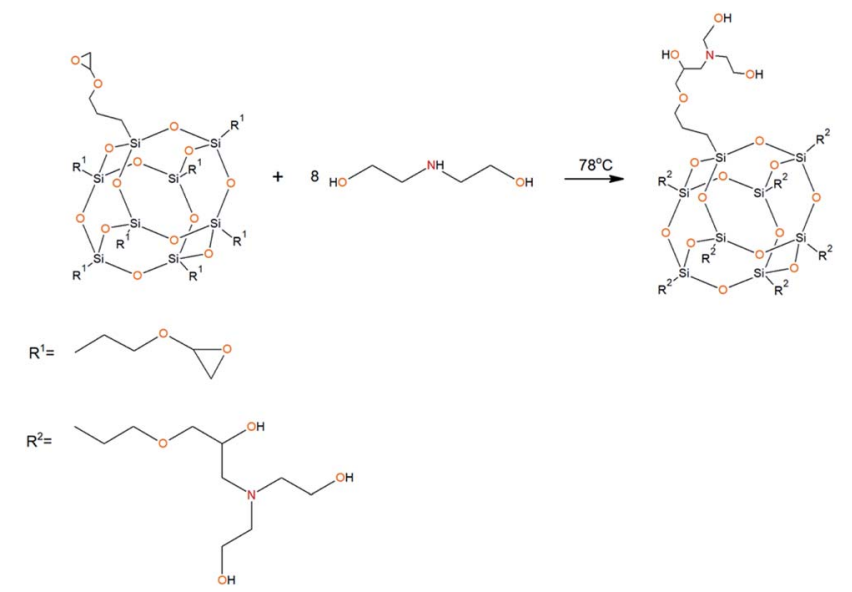

Scheme 5 Synthesis of DEA-Gl-POSS.

$40{ }^{\circ} \mathrm{C} / 22{ }^{\circ} \mathrm{C}$ of WVP for AEBA-PEG-PU is 2.5 ; for AEBA-EMD-2.25$\mathrm{PU}$ it reaches 6.2; for AEBA-EMD-1.5-PU it is 5.4.

Studies of mechanical properties (Fig. 4 and Table 2) showed that the strength and the Young modulus of AEBA-EMD-PU do not change additively with an increase of EMD content, but instead there are areas of a fall followed by a subsequent increase. Such a phenomenon is usually associated with the hierarchy of the supramolecular structure. That is, small quantities form the primary structure, in which the elements of the macromolecule form additional intermolecular bonds. The increase in the content of EMD changes the ways of constructing such structures.

According to measurements of TMA and DMA (Fig. 5), the glass transition temperature of AEBA-EMD-1.0-PU is in the region of 

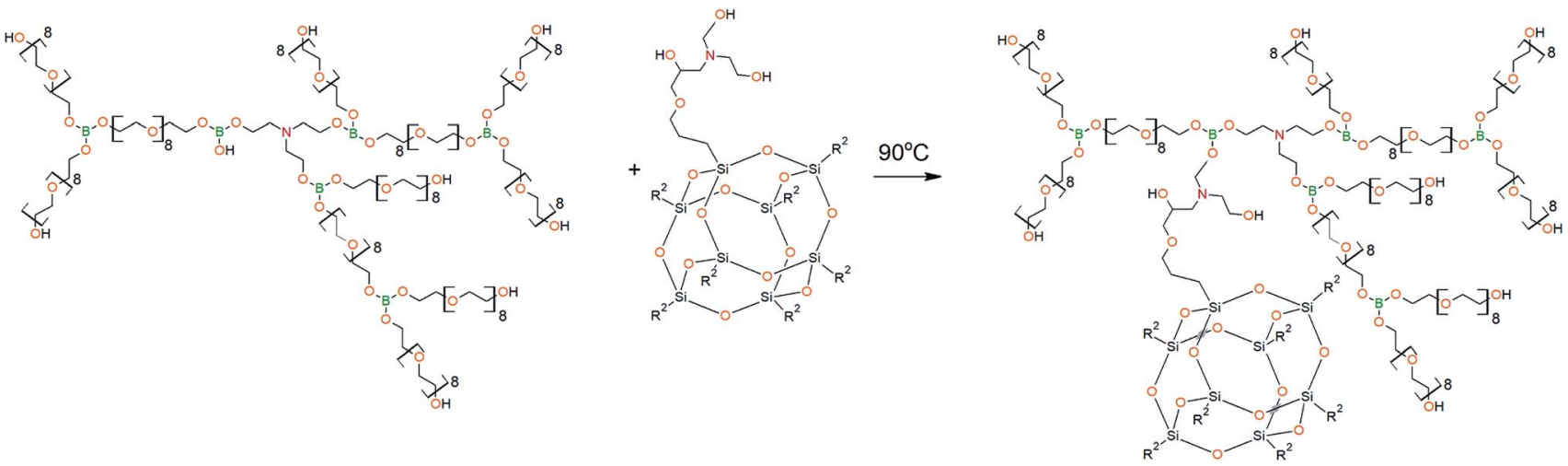

Scheme 6 Synthesis of AEBA-DEA-Gl-POSS.

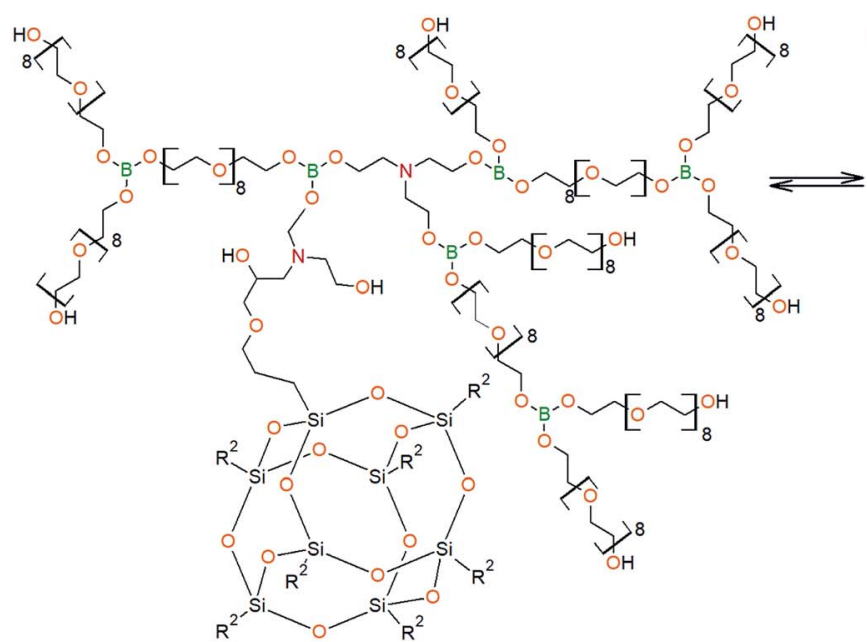

where $\int$ - is an anion-forming bond

with a neighboring molecule

Scheme 7 Scheme of intermolecular interaction and complex formation in AEBA-DEA-GL-POSS.

$25{ }^{\circ} \mathrm{C}$. With a further increase in the content of EMD in the composition of AEBA-EMD, the glass transition temperature reaches $40{ }^{\circ} \mathrm{C}$.

An important conclusion of the studies performed is the lack of correlation between the glass transition temperature and the WVP values for AEBA-EMD-PU.

In this way, hard aromatic block, which is a structural element of diglycidyl ether of 4,4'-dihydroxy-2,2-diphenylpropane in EMD, plays a key role in the formation of the supramolecular structure of PUs based on AEBA-EMD, creating ordering zones in bulk of the matrix. Segregation of rigid blocks is accompanied by the forced unification of the short-chain poly(oxyethylene glycol) component. All these described processes of formation of a supramolecular structure lead to an increase in free volume in such polyurethanes.

To create steric hindrances in the hyperbranched structure of aminoethers of boric acid one more adduct on the basis of polyhedral octaglycidyl-silsesquioxane (Gl-POSS) and diethanolamine (DEA) was synthesized. Such an adduct (DEA-Gl-POSS) does not contain symmetric rigid blocks, and it cannot segregate in ordering zones in bulk matrices. The possibility of including DEA-Gl-POSS in AEBA-PEG is determined by the molar ratio of $[\mathrm{TEA}]:\left[\mathrm{H}_{3} \mathrm{BO}_{3}\right]:[\mathrm{PEG}]=1: 6: 11$. At this molar ratio, one $\mathrm{B}-\mathrm{OH}$ group remains and can be involved in the etherification reaction with terminal hydroxyl groups of DEA-Gl-POSS.

\subsection{Synthesis and characterization of amino ethers of boric acid modified by adduct DEA-Gl-POSS}

The DEA-Gl-POSS synthesis is shown in Scheme 5 and the AEBADEA-Gl-POSS synthesis is presented in Scheme 6.

Similarly to AEBA-EMD, the values of dynamic viscosity of AEBA-DEA-Gl-POSS were also observed to significantly exceed the

Table 3 Density of AEBA-DEA-Gl-POSS

\begin{tabular}{lc}
\hline DEA-Gl-POSS content, wt $\%$ & $\rho, \mathrm{g} \mathrm{cm}^{-3}$ \\
\hline 0 & 1.1434 \\
0.5 & 1.1528 \\
0.7 & 1.1434 \\
0.8 & 1.1513 \\
1 & 1.1512
\end{tabular}




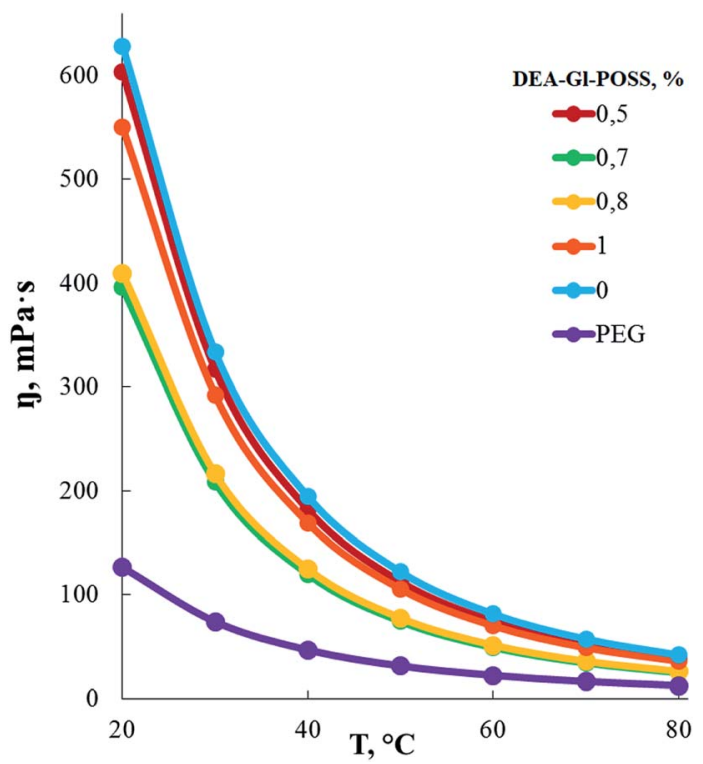

Fig. 6 Dynamic viscosity of AEBA-Gl-POSS.

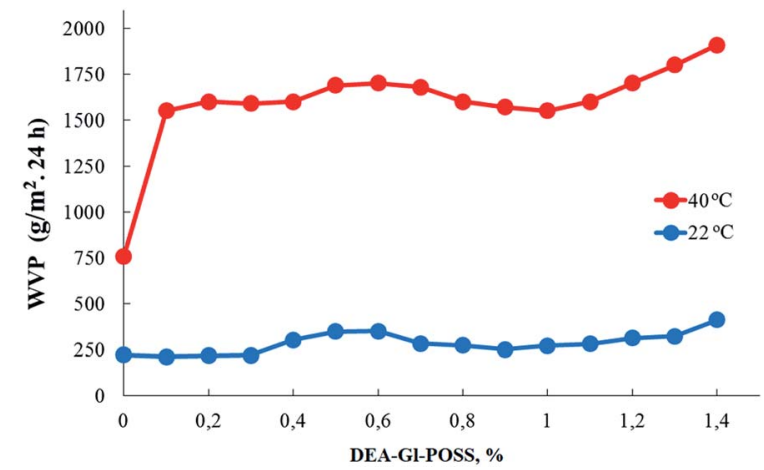

Fig. 7 Dependence of WVP for AEBA-DEA-GL-POSS-PU on the content of DEA-GI-POSS.

values obtained for PEG. However, unlike AEBA-EMD, in the case of AEBA-DEA-Gl-POSS, the values of dynamic viscosity vary with the content of DEA-Gl-POSS in the composition of AEBA-DEA-GlPOSS. This can be explained by the fact that DEA-Gl-POSS creates significant steric hindrances in the formation of intermolecular complexes in case of AEBA-DEA-Gl-POSS. This means that AEBADEA-Gl-POSS is also involved in the formation of intermolecular complexes, accompanied by the formation of borate anions and tertiary ammonium cations according to Scheme 7 .

According to the results of density measurements for AEBADEA-Gl-POSS (Table 3), the introduction of large hydroxylterminated molecules of DEA-Gl-POSS into the AEBA-PEG structure similarly to AEBA-EMD leads to changes in the spatial packaging of hyperbranched aminoethers of boric acid (Fig. 6).

\subsection{PU films based on hyperbranched amino ethers of boric acid modified by adduct DEA-Gl-POSS}

Film samples of AEBA-DEA-Gl-POSS-PU were tested for water vapor permeability (Fig. 7). The data obtained indicate a high

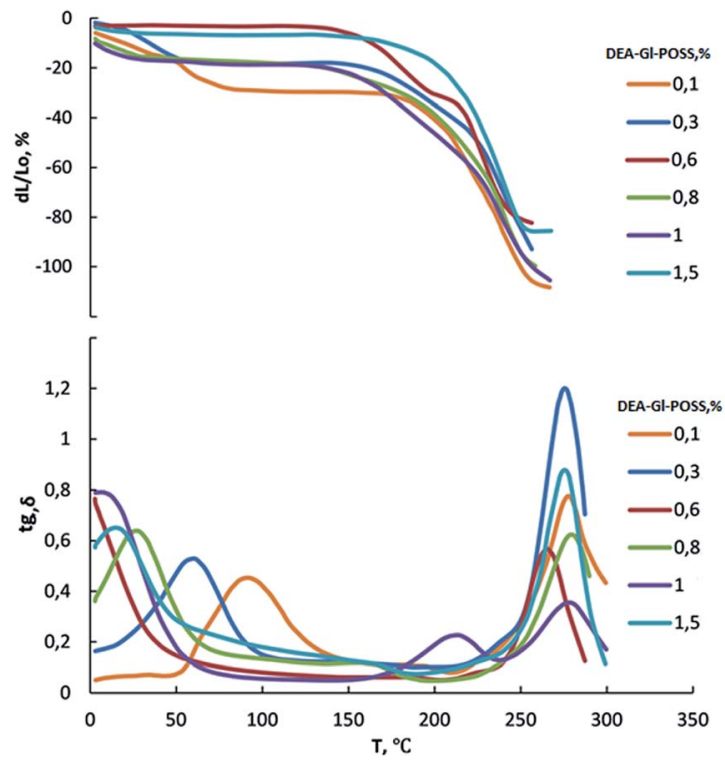

Fig. 8 TMA and MLT curves of AEBA-DEA-GL-POSS-PU.

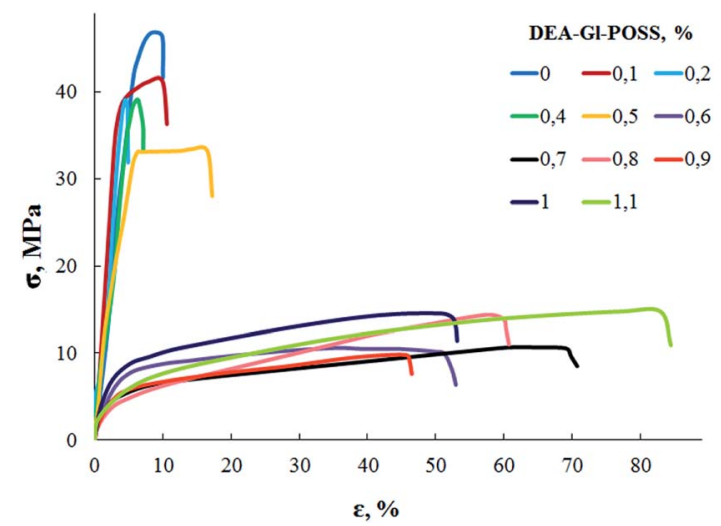

Fig. 9 Tensile tests of AEBA-DEA-GL-POSS-PU.

WVP of the AEBA-DEA-Gl-POSS-PU film polymeric materials obtained. Similarly to AEBA-EMD-PU (Fig. 3), the values of WVP at $22{ }^{\circ} \mathrm{C}$ are little dependent on the EMD content. With an increase in temperature to $40{ }^{\circ} \mathrm{C}$, a noticeable increase in the values of WVP is observed. The use of DEA-Gl-POSS for the

Table 4 Young's modulus for AEBA-DEA-GI-POSS-PU with different content of DEA-GL-POSS

\begin{tabular}{lc} 
DEA-Gl-POSS content, wt $\%$ & Young's modulus, MPa \\
\hline 0 & 898 \\
0.1 & 890 \\
0.2 & 717 \\
0.4 & 1233 \\
0.5 & 1028 \\
0.6 & 351 \\
0.7 & 356 \\
0.8 & 489 \\
0.9 & 594 \\
1 & 567 \\
1.1 & 481
\end{tabular}


synthesis of the corresponding PU films leads to a twofold increase in WVP compared to unmodified AEBA-PEG-PU. For AEBA-DEA-Gl-POSS-1.4-PU, WVP can reach values of $1880 \mathrm{~g} \mathrm{~m}^{-2}$ for 24 hours at $40{ }^{\circ} \mathrm{C}$. The ratio $40{ }^{\circ} \mathrm{C} / 22{ }^{\circ} \mathrm{C}$ of WVP for AEBAPEG-PU is 2.5; for AEBA-DEA-Gl-POSS-0.3-PU it reaches 7.6; for AEBA-DEA-Gl-POSS-1.4-PU it is 4.6.

According to Fig. 8, the thermomechanical behavior of AEBADEA-Gl-POSS-PU samples as well as their vapor permeability is hierarchical. In general, polymers exhibit high heat resistance, reaching $240{ }^{\circ} \mathrm{C}$. It turned out that the temperature of the onset of segmental mobility depends on the content of DEA-Gl-POSS in the composition of AEBA-DEA-Gl-POSS. Thus, at $0.1 \mathrm{wt} \%$ of DEA-Gl-POSS, the highest temperature of the $\alpha$-transition (80 ${ }^{\circ} \mathrm{C}$ ) is observed, which decreases with an increase in content of the adduct to $0{ }^{\circ} \mathrm{C}$. According to the changes in the thermomechanical behavior of the samples, their mechanical behavior also changes. So, with the growth of the percentage of DEA-GlPOSS up to $0.5 \mathrm{wt} \%$, samples of AEBA-DEA-Gl-POSS-PU retain relatively high values of the strength and Young's modulus (Fig. 9 and Table 4). With a further increase in the content of DEA-Gl-POSS above $0.6 \mathrm{wt} \%$, a distinctive feature of the samples is a relatively low values of the strength and Young's modulus and a relatively high values of stretching.

It is important to emphasize that vapor permeability values are in no way related to the glass transition temperature. That is, vapor permeability has no regular connection between the glass transition temperature and vapor permeability values.

Thus, the results of the research allow us to conclude that the vapor permeability of PU films obtained on the basis of sterically hindered aminoethers of boric acid is due to the ionomeric nature of AEBA-PU and the presence of steric hindrances in the hyperbranched AEBA, which can lead to an increase in free volume in such PUs.

High values of WVP and the ratio of $40{ }^{\circ} \mathrm{C} / 22{ }^{\circ} \mathrm{C}$ of WVP, heat resistance and peculiarity of mechanical behavior of obtained polyurethanes provide the possibilities of their application for the manufacture of protective clothings, leather or textile finishing.

\section{Conclusions}

On the basis of diglycidyl ether of 4,4'-dihydroxy-2,2diphenylpropane, diethanolamine and monoethanolamine, hydroxyl-terminated adducts of EMD were synthesized. EMD were used to modify the chemical structure of the hyperbranched aminoethers of boric acid (AEBA-PEG) and to obtain the corresponding AEBA-EMD.

Similarly, the corresponding AEBA-DEA-Gl-POSS was synthesized using the adduct (DEA-Gl-POSS) based on the onestep reaction of octaglycidyl polyhedral oligomeric silsesquioxane and diethanolamine.

The peculiarity of AEBA-EMD and AEBA-DEA-Gl-POSS is the presence of spatially separated ion pairs in there chemical structure as a result of the processes of intermolecular complexation.

Changes in the content of EMD in AEBA-EMD and DEA-GlPOSS in AEBA-DEA-Gl-POSS have a significant impact on the properties of polyurethanes, obtained on their basis. The hierarchical nature of changes in mechanical behavior of polyurethanes due to the content of adducts indicates the ambiguous nature of their influence on the macromolecular organization of polyurethanes.

It was shown that the processes of water diffusion through the polyurethane films obtained on the basis of sterically hindered aminoethers of boric acid are due to the peculiarities of the chemical structure of AEBA, which can exhibit ionomeric nature and the presence of steric hindrances created in the hyperbranched structure of AEBA, which can lead to an increase in free volume in such polyurethanes.

\section{Conflicts of interest}

There are no conflicts to declare.

\section{Abbreviation}

HBPs

AEBA

TEA

PEG

DEA

MEA

Gl-POSS

AEBA-PEG

ED-20

EMD

DEA-Gl-POSS

AEBA-EMD

AEBA-DEA-Gl-

POSS

PIC

PU

AEBA-EMD-PU

AEBA-DEA-Gl-

POSS-PU

WVP
Hyperbranched polymers

Amino ethers of boric acid

Triethanolamine

Poly(oxyethylene glycol) with the number of oxyethylene units equal to 9

Diethanolamine

Monoethanolamine

Octaglycidyl polyhedral oligomeric silsesquioxane

Amino-ethers based on TEA, boric acid and PEG

Diglycidyl ether of 4,4'-dihydroxy-2,2diphenylpropane

The adduct based on the one-step reaction of ED-20, MEA and DEA

The adduct based on the one-step reaction of Gl-POSS and DEA

AEBA-PEG, modified by EMD

AEBA-PEG, modified by Gl-POSS

Polyisocyanate "Wannate PM-200"

Polyurethane

Polyurethanes based on AEBA-EMD and PIC

Polyurethanes based on AEBA-DEA-Gl-POSS

and PIC

Water vapor permeability

\section{Acknowledgements}

This work was supported by the Russian Science Foundation (grant no. 19-19-00136).

\section{References}

1 I. Yilgor and E. Yilgor, Polymer, 1999, 40, 5575.

2 V. Bharadwaj, K. Somani and S. Kansara, J. Macromol. Sci., Part A: Pure Appl.Chem., 2002, 39, 115.

3 W. Chen, C. Zhu and X. Gu, J. Appl. Polym. Sci., 2002, 84, 1504. 
4 S. Hayashi, N. Ishikawa and C. Giordano, J. Coated Fabr., 1933, 23, 74.

5 J.-L. Hu and S. Mondal, Polym. Int., 2005, 54(5), 764.

6 A. Morel, F. Salau, G. Bedek, D. Dupont and S. Giraud, J. Mater. Sci., 2016, 52, 1014.

7 K. Y. Hsieh, C. C. Tsai and S. M. Tseng, J. Membr. Sci., 1990, 49, 341.

8 J. Leng, X. Lan, Y. Liu and S. Du, Prog. Mater. Sci., 2011, 56, 1077.

9 Y. Chen, Y. Liu, H. Fan, H. Li, B. Shi, H. Zhou and B. Peng, J. Membr. Sci., 2007, 287, 192.

10 S. Mondal and J. L. Hu, Carbohydr. Polym., 2007, 67, 282.

11 S. Mondal, J. L. Hu and Z. Yong, J. Membr. Sci., 2006, 280, 427.

12 H. M. Jeon, B. K. Ahn and B. K. Kim, Polym. Int., 2000, 49, 1714.

13 H. M. Jeong, B. K. Ahn, S. M. Cho and B. K. Kim, J. Polym. Sci., Part B: Polym. Phys., 2000, 38, 3009.

14 H. Shi, Y. Chen, H. Fan, J. Xiang and B. Shi, J. Appl. Polym. Sci., 2010, 117, 1820.

15 P. J. Flory, J. Am. Chem. Soc., 1952, 74, 2718.

16 K. Kirkorian, A. Ellis and L. J. Twyman, Chem. Soc. Rev., 2012, 61, 6138.

17 Y. Zheng, S. Li, Z. Weng and C. Gao, Chem. Soc. Rev., 2015, 44, 4091.

18 D. Hu, S. Jin, Y. Shi, X. Wang, R. W. Graff, W. Liu, M. Zhu and H. Gao, Nanoscale, 2017, 9, 3629.

19 C.-Q. Li, F.-F. Wang, R. Gao, P. Sun, N. Zhang and J. Wang, Transition Met. Chem., 2017, 42, 339.

20 N. Zhang, S. Wang, L. Song, C. Li and J. Wang, Chem. Pap., 2016, 71, 1037.

21 Y. Shi, L. Liu, F. Zhang, M. Niu, Y. Zhao, Y. Fan, Y. Liang, M. Liu, Z. Zhang and J. Wang, Polymers, 2017, 9, 459.

22 X. Huang, A. Varyambath, M. R. Kim, M. Kim and I. Kim, J. Nanosci. Nanotechnol., 2017, 17, 7668.

23 N. Zhang, J. Wang, H. Huo, L. Chen, W. Shi, C. Li and J. Wang, Inorg. Chim. Acta, 2018, 469, 209.

24 J. Li and Z. S. Bo, Macromolecules, 2004, 37, 2013.

25 K. Kim, H. B. Lee, J. W. Lee, H. K. Park and K. S. Shin, Langmuir, 2008, 24, 7178.

26 J.-J. Feng, L. Liu, H. Huang and A.-J. Wang, Sens. Actuators, B, 2017, 238, 91.

27 M. S. Selim, F. Q. Wang, H. Yang, Y. Huang and S. Kuga, Mater. Des., 2017, 135, 173.

28 M. Keramatinia, F. Najafi and M. R. Saeb, Prog. Org. Coat., 2017, 113, 151.

29 Q. Ban, H. Chen, Y. Yan, N. Tian and J. Kong, Eur. Polym. J., 2017, 96, 474.

30 G. Gogoi, S. Gogoi and N. Karak, Prog. Org. Coat., 2017, 112, 57.

31 B. Gao, J. Zhang, Z. Hao, L. Huo, R. Zhang and L. Shao, Carbon, 2017, 123, 548.

32 Y. Harinath, D. Harikishore Kumar Reddy, L. Subramanyam Sharma and K. Seshaiah, J. Environ. Chem. Eng., 2017, 5, 4994.

33 I. M. Davletbaeva, O. O. Sazonov, A. R. Fazlyev, R. S. Davletbaev, S. V. Efimov and V. V. Klochkov, RSC Adv., 2019, 9, 18599.
34 Q. Jin, Y. Wang, T. Cai, H. Wang and J. Ji, Polymer, 2014, 55, 4641.

35 I. Mamajanov, M. P. Callahan, J. P. Dworkin and G. D. Cody, Origins Life Evol. Biospheres, 2015, 45, 123.

36 B. Zhang, J. Wang, J. Chen, H. Zhang, D. Yin and Q. Zhang, Biochem. Eng. J., 2017, 127, 43.

37 L. Zhang, Y. Zhou, G. Shi, X. Sang and C. Ni, Mater. Sci. Eng., C, 2017, 79, 116.

38 A. K. Pearce, J. D. Simpson, N. L. Fletcher, Z. H. Houston, A. V. Fuchs, P. J. Russell, A. K. Whittaker and K. J. Thurecht, Biomaterials, 2017, 141, 330.

39 W. Wang, J. Ma, F. Jin and J. Liao, Exp. Ther. Med., 2017, 14(4), 3105.

40 D. Yan, C. Gao and H. Frey, Hyperbranched Polymers. Synthesis, Properties, and Applications, John Wiley \& Sons, Hoboken, 2011, p. 480.

41 J. Fang, H. Kita and K. Okamoto, Macromolecules, 2000, 33, 4639.

42 Y. Yampolskii and B. Freeman, Membrane Gas Separation, John Wiley \& Sons, Chichester, 2010, p. 392.

43 A. Rybak, A. Rybak, W. Kaszuwara, S. Awietjan, P. Sysel and Z. J. Grzywna, Mater. Lett., 2017, 208, 14.

44 H. He, Y. Hu, S. Chen, L. Zhuang, B. Ma and Q. Wu, Sci. Rep., 2017, 7, 3913.

45 Z. Yang, Q. Wang and T. Wang, J. Mater. Chem. A, 2017, 5, 13823.

46 T. Suzuki, M. Takenaka and Y. Yamada, J. Membr. Sci., 2017, $521,10$.

47 H. Yao, N. Zhang, K. Shen, N. Song, K. Shi, S. Zhu, Y. Zhang and S. Guan, Polymer, 2017, 115, 176.

48 T. Gurunathan, S. Mohanty and S. K. Nayak, Polym.-Plast. Technol. Eng., 2015, 55, 92.

49 J. Mulder, Basic Principles of Membrane Technology, Academic Publishers, Dordrecht, 2000, p. 565.

50 T. Suzuki, Y. Yamada and Y. Tsujita, Polymer, 2004, 45, 7167.

51 L. Shao, T. Chung, S. Goh and K. Pramoda, J. Membr. Sci., 2004, 238, 153.

52 A. S. Kovvali and K. K. Sirkar, Ind. Eng. Chem. Res., 2002, 41, 2287.

53 T. Suzuki, Y. Yamada and K. Itahashi, J. Appl. Polym. Sci., 2008, 109, 813.

54 K. Johansson, T. Bergman and M. Johansson, ACS Appl. Mater. Interfaces, 2009, 1, 211.

55 H. J. Jeong and B. K. Kim, React. Funct. Polym., 2017, 116, 92.

56 I. M. Davletbaeva, O. Yu. Emelina, I. V. Vorotyntsev, R. S. Davletbaev, E. S. Grebennikova, A. N. Petukhov, A. I. Akhmetshina, T. S. Sazanova and V. V. Loskutov, RSC Adv., 2015, 5, 65674.

57 I. M. Davletbaeva, G. R. Nurgaliyeva, A. I. Akhmetshina, R. S. Davletbaev, A. A. Atlaskin, T. S. Sazanova, S. V. Efimov, V. V. Klochkov and I. V. Vorotyntsev, RSC $A d v .$, 2016, 6, 111109.

58 E. Malmstrom and A. Hult, J. Macromol. Sci., Rev. Macromol. Chem. Phys., 1997, 37, 555.

59 B. Romagnoli and W. Hayes, J. Mater. Chem., 2002, 12, 767. 